- admits that he was surprised that his field was picked ahead of gravitational-wave astronomy, given that in previous competitions, including the US Decadal Survey, which decides future NASA missions, the latter had been given the edge.

Many astrophysicists think that the ideal situation would be to go for both projects in parallel, because the two observatories would be looking at some of the same targets, such as black holes, albeit through very different eyes. Bringing the gravitational mission forward would require a cash injection. But Danzmann says that China has already expressed an interest in getting involved with a space-based gravitational-wave detector. And NASA, which pulled out of a similar joint mission in 2011, might be able to reconsider contributing after the launch of the James Webb Space Telescope in 2018, he suggests.

ESA's science programme schedules three missions of this scale every 20 years (see ' $\mathrm{X}$-ray

\title{
X-RAY SPECS
}

The European Space Agency is very close to finalizing the following line-up of large (L-class) missions.

\begin{tabular}{l|l|l|l|} 
Mission & Name & Launch date & Study targets \\
\hline L1 & JUICE & 2022 & The magnetic field of Jupiter's moon Ganymede \\
L2 & Athena+ & 2028 & Black holes and galaxy clusters. \\
L3 & eLISA & 2034 & Gravitational ripples from black-hole mergers.
\end{tabular}

specs'). In May last year, the Jupiter Icy Moons Explorer (JUICE) was approved as the first of three missions under ESA's Cosmic Vision programme, intended for the L1 launch opportunity in 2022. At that time, previous incarnations of Athena+ and eLISA missed out on selection. The L2 and L3 science themes were based on recommendations from ESA's independent Senior Survey Committee, chaired by astronomer Catherine Cesarsky, a former directorgeneral of the European Southern Observatory.

The current two winners were selected from 32 proposals presented at a meeting in
September. Other options considered were a $\gamma$-ray telescope, a mission to explore interstellar space and a host of proposals to explore moons and planets in the Solar System.

Although disappointed that no planetary missions have been put forward, Colin Wilson, a planetary physicist at the University of Oxford, UK, says that his field will still be busy in the coming decade, through planned ESA missions to Mercury, Mars and Jupiter.

But, he adds, exploring the farthest realms of the Solar System, beyond Saturn, will be a real challenge in the coming decades.

\section{Precision gene editing paves way for transgenic monkeys}

\section{Despite political challenges, engineered primates could be better disease models than mice.}

\section{BY HELEN SHEN}

A nthony Chan spent two years creating the first five monkeys in the world to be genetically engineered with human mutations - in this case, for Huntington's disease. But three of the five monkeys, reported in 2008, developed severe symptoms of Huntington's much more quickly than anticipated, and had to be killed within a month of birth ${ }^{1}$. Viruses used to introduce the relevant gene had inserted extra copies randomly, intensifying the symptoms - and highlighting the method's limitations in creating animal disease models.

Chan, a geneticist at Emory University in Atlanta, Georgia, and other scientists around the world are now eyeing precision genomeediting techniques that solve such problems by using enzymes and RNA instead of viruses. Many have high hopes that transgenic monkeys will mimic human genetic conditions more faithfully than mice - and thus permit better drug-development tests. Many also say

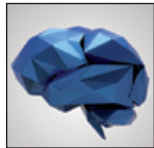

NEW ANGLES ON THE BRAIN

A Nature special issue

www.nature.com/neuroscience2013

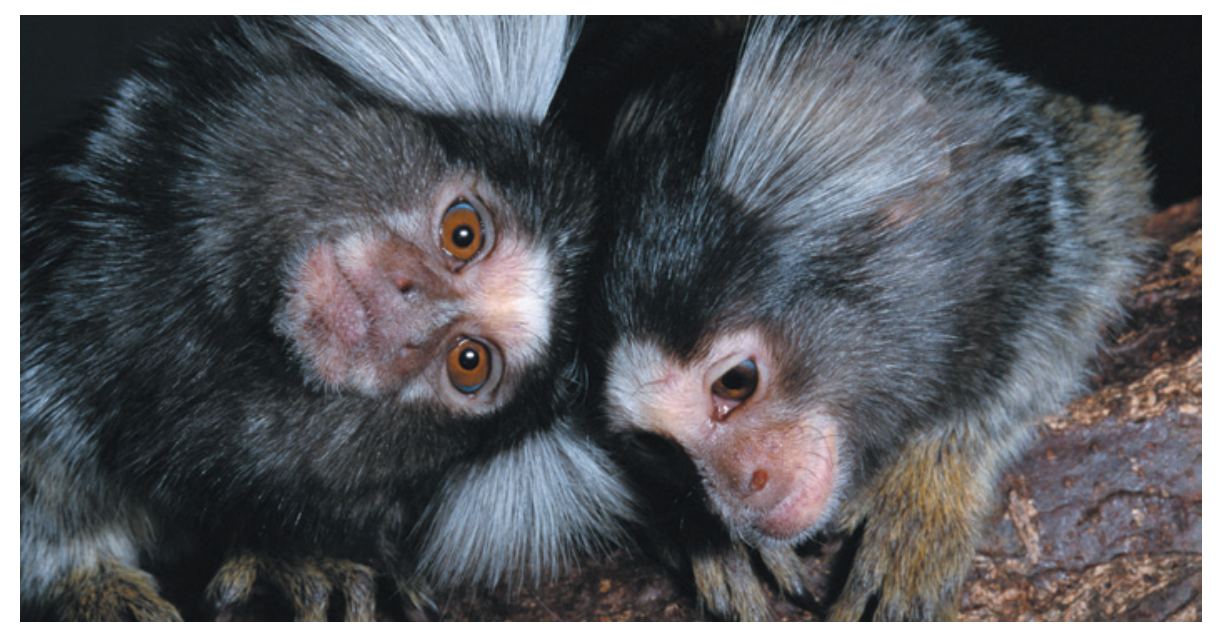

Marmosets are among the primates that may soon be engineered with custom genetic-editing methods.

that the primates will accelerate basic research in neuroscience, permitting researchers to map and test complex neural circuits underlying behaviours that do not exist in simpler organisms. "We weren't even able to think about this before," says Chan.

Work is already under way in a few labs. Erika Sasaki, a geneticist at Keio University in Tokyo, was part of the team that used viruses to produce the first transgenic marmoset to pass on an engineered gene to its offspring ${ }^{2}$. On 11 November, at the annual meeting of the Society for Neuroscience in San Diego, California, Sasaki and her colleagues will present efforts to model autistic behaviour in marmosets using mutations produced with zincfinger nucleases - a class of DNA-cutting enzyme. Next month, the Salk Institute for 


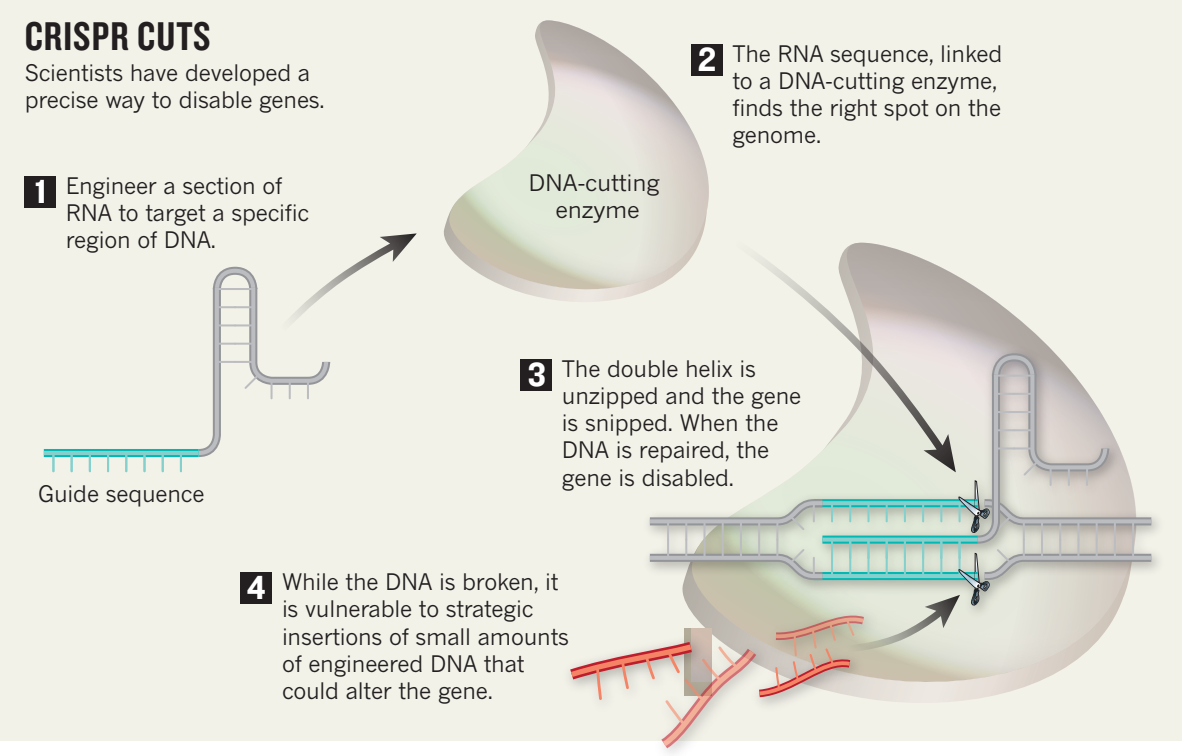

Biological Studies in La Jolla, California, is hosting a workshop to bring together molecular biologists, bioengineers and neuroscientists to work on challenges in the field. "This is something with tremendous potential," says Terrence Sejnowski, head of the computational neurobiology laboratory at the Salk Institute.

Neuroscientists have long wanted transgenic monkeys. Disorders such as autism, schizophrenia and Alzheimer's disease cannot be fully replicated in mice, which lack the complex cognitive and social abilities of primates. Moreover, many neuroactive drugs that showed early promise in mice have failed in human trials.

But researchers have persisted in working with mice, mainly because there is a targeted gene-editing strategy that works in the animals. It relies on extremely rare, spontaneous DNA-swapping events to alter or disable certain genes. The method requires several arduous steps: designing the desired mutation and introducing it to embryonic stem cells; detecting it in the tiny fraction of stem cells that incorporate it; integrating those stem cells into a developing mouse embryo; and then hoping that when the 'patchwork' animal matures, its sperm or eggs will carry the mutation, allowing the creation of transgenic offspring.

The approach is feasible only because mouse stem cells are relatively cheap to maintain and screen, and because mice reach sexual maturity within weeks and produce large litters.
"You don't have that luxury with monkeys," says Edward Callaway, a neuroscientist at the 15 months to mature sexually, carry babies for 5 months and often give birth to only 2 young. Macaques take even longer -3 years to reach sexual maturity, and they typically produce a single baby after a 5.5-month gestation.

But custom monkeys have become a possibility thanks to efficient gene-editing techniques that can support a method in which embryos are manipulated one at a time. In one method, zinc-finger nucleases home in on specific genomic regions, and cut genes open to disrupt their function or allow researchers to substitute in external DNA. In another, called CRISPR, a customizable RNA fragment is used to guide a DNA-cutting enzyme to the right spot (see 'CRISPR cuts'). Feng Zhang, a synthetic biologist at the Massachusetts Institute of Technology (MIT) in Cambridge, showed in May that CRISPR could be used to make precise mutations in multiple genes in mouse embryos ${ }^{3}$. That, he says, could pave the way for monkey models of human brain disorders that involve more than one gene.

"We now have, for the first time, the possibility of trying some of the treatments for genetic-based diseases in primates," says Robert Desimone, director of the McGovern Institute for Brain Research at MIT.

MIT researchers are now testing CRISPR on Salk Institute. Marmosets, for example, take fertilized monkey eggs, in collaboration with researchers from the Oregon National Primate Research Center in Beaverton. They will start by disabling genes. MIT neuroscientist Guoping Feng, one of Zhang's collaborators, wants to disrupt a gene called SHANK3, which has been implicated in some human cases of autism. More sophisticated changes, such as swapping in a different version of a gene, will require more work and engineering, says Zhang.

Eventually, he adds, CRISPR might be used to label specific monkey neuron types or control them with light - just as is currently possible with mouse neurons. Basic neuroscientists are especially keen to see those applications succeed. Anthony Movshon, a monkey-vision researcher at New York University, has watched over the past decade as scientists have used genetic tools to stimulate, inhibit and record neural activity in mice and flies. But for many important areas of neuroscience - cognition, attention, memory and decision-making those animals are "not that interesting", he says.

All of this excitement comes at a time when monkey research has become increasingly difficult. In January, following years of pressure by
"We now have, for the first time, the possibility of trying some of the treatments for geneticbased diseases in primates." animal-rights activists, United Airlines announced that it would stop transporting research monkeys - eliminating the last North American air carrier available to primate researchers (see Nature 483, 381-382; 2012). And in April, the New England Primate Research Center in Southborough, Massachusetts - one of eight such centres supported by the US National Institutes of Health - announced that it would close after gradually transferring its animals to other facilities.

Despite the controversies, some researchers say that using transgenic monkeys may be the best way to search for treatments for brain diseases and to investigate how networks of neurons create consciousness. "You want to match the model system to the question you're asking," says Movshon. "It's just as unethical to use a mouse for an experiment for which it's not suited."

1. Yang, S.-H. et al. Nature 453, 921-924 (2008).

2. Sasaki, E. et al. Nature 459, 523-527 (2009).

3. Wang, H. et al. Cell 153, 910-918 (2013).

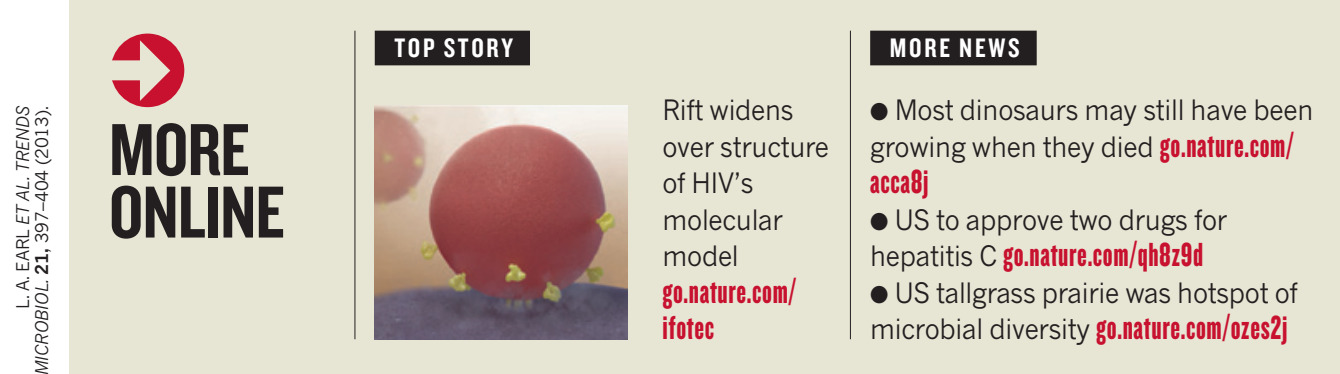

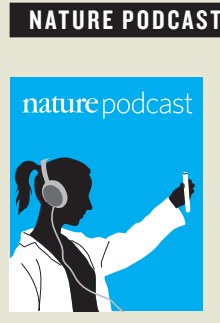

Eye contact in infants predicts autism; a fresh take on Parkinson's; and real science in schools nature.com/ nature/podcast 